\title{
CORRELAÇÃO ESPACIAL E LINEAR DE ATRIBUTOS FÍSICOS DO SOLO EM SISTEMA DE INTEGRAÇÃO LAVOURA PECUÁRIA ${ }^{1}$
}

\author{
Danilo Gomes de Oliveira ${ }^{*}$, Elton Fialho dos Reis ${ }^{2}$, João Carlos Medeiros ${ }^{3}$, Rodney Ferreira Couto ${ }^{4}$, \\ Vandoir Holtz ${ }^{5}$ Beata Emoke Madari ${ }^{6}$
}

\author{
${ }^{\text {"Eng }}{ }^{0}$ Agrícola, Prof. Mestre, Instituto Federal do Tocantins - IFTO/ Campus Colinas do Tocantins -TO; \\ ${ }^{2}{ }^{E} n{ }^{\circ}$ Agrícola, Prof. Doutor, Universidade Estadual de Goiás - UEG/Campus Anápolis- GO; \\ ${ }^{3} E_{n g}{ }^{0}$ Agrônomo, Prof. Doutor, Universidade Federal do Piauí -CPCE/UFPI-PI; \\ ${ }^{4}$ Eng ${ }^{\circ}$ Agrícola, Prof. Mestre, Universidade Estadual de Goiás- UEG/Campus Anápolis-GO; \\ ${ }^{5} E_{n g}{ }^{0}$ Agrícola, Prof. Mestre, Universidade Estadual do Mato Grosso, UNEMAT/Campus Nova Xavantina - MT; \\ ${ }^{6}$ Eng ${ }^{\circ}$ Agrônomo, Embrapa Arroz e Feijão, Santo Antônio de Goiás, Goiás, Brasil; \\ *Autor para correspondência: Danilo Gomes de Oliveira, danilogomes.engenharia@gmail.com.
}

\begin{abstract}
RESUMO: O sistema de Integração lavoura-pecuária (iLP) baseia-se na diversificação da produção agrícola nas propriedades rurais. Constitui-se da rotação planejada das atividades agrícolas e pecuárias em uma mesma área, de forma a beneficiar ambas. $O$ objetivo deste trabalho foi avaliar a variabilidade espacial e a correlação de atributos físicos do solo no sistema de iLP. Para isso, foi construído um grid amostral regular de $25 \times 25 \mathrm{~m}$, totalizando 50 pontos em uma área de $7 \mathrm{ha}$, com dois tipos de cobertura. Os pontos foram georeferenciados e em seguida realizadas as coletas de amostras indeformadas de solo em duas camadas: P1 (0,00-0,10 m) e P2 (0,20-0,30 m). Foram mensurados os atributos: umidade gravimétrica do solo $(\mathrm{Ug})$, densidade (Ds) e resistência à penetração (RP). Para a análise estatística descritiva, correlações espaciais e a construção dos mapas de krigagem e cokrigagem foi empregado o software GS+ versão 7.0. As correlações lineares foram feitas no software $R$ versão 3.1.1. A correlação linear entre os atributos físicos avaliados foi classificada como fraca. A correlação espacial e os mapas de krigagem dos atributos apresentaram um índice de dependência espacial classificado como fracas, explicadas pelos modelos gaussiano, exponencial e esférico.
\end{abstract}

PALAVRAS-CHAVE: agricultura de precisão; geoestatística, dependência espacial.

\section{SPATIAL VARIABILITY OF PHYSICAL ATTRIBUTES OF THE SOIL IN LIVESTOCK FARMING SYSTEM INTEGRATION}

ABSTRACT: The integrated crop-livestock system (ILP) is considered more productive and sustainable because it encourages diversification, rotation of agricultural activities and livestock within the farm, providing benefits to both. The objective of this study was to evaluate the spatial variability and the correlation of soil physical properties in the ILP system. For that a regular sampling grid of $25 \mathrm{~m} \times 25 \mathrm{~m}$ was created, totaling 50 points in an area of 7 ha, with two types of coverage. The sampling points were georeferenced. Undisturbed samples were taken in each at two depths: $\mathrm{P} 1(0.00$ to $0.10 \mathrm{~m})$ and $\mathrm{P} 2(0.20$ to $0.30 \mathrm{~m})$. Soil moisture, bulk density and resistance to penetration were measured. For descriptive statistical analysis, spatial correlations and the construction of kriging maps using the GS + version 7.0 software. Linear correlations were made using the $R$ version 3.1.1 software. The linear correlation between the assessed physical attributes were classified as poor. The spatial correlation and kriging maps of the attributes presented a spatial dependence index classified as weak, explained by Gaussian models, exponential and spherical.

KEYWORDS: precision agriculture, geostatistics, spatial dependence.

\section{INTRODUÇÃO}

A utilização de sistemas integrados de produção, como o iLP, caracteriza-se pela alternância entre a produção de grãos (agricultura) e pasto para os animais (pecuária). Fundamenta-se na integração dos componentes do sistema produtivo, busca sempre 
elevar a qualidade do que é produzido, preservar os recursos naturais e manter produtividade satisfatória (Kunz et al., 2013). Portanto, apresenta-se como uma estratégia para maximizar efeitos desejáveis no ambiente, aliado ao aumento da produtividade das culturas com a melhoria da qualidade das pastagens. Principalmente, quando é desenvolvido seguindo seus fundamentos básicos. Em muitos casos, pode apresentar produção superior à dos sistemas constituídos somente com o cultivo exclusivo (Nicoloso et al., 2006; Costa et al., 2015).

Uma das principais causas da degradação do solo é a compactação, resultado do processo de aumento da densidade e da resistência à penetração das raízes (Maluf et al., 2012). É frequentemente observada em áreas de agricultura onde se utiliza máquinas e implementos pesados ou em áreas de pisoteio de animais intenso, constituindo um dos fatores de restrição ao desenvolvimento das plantas nestes ambientes (Souza et al., 2012; Cortez et al., 2014).

Nos sistemas de iLP, uma das causas da compactação do solo é o pisoteio animal, provocada pelas altas lotações e elevada pressão de pastejo, especialmente em períodos úmidos. Situação que é mais crítica para os solos argilosos que, por possuírem menos macroporos que os solos de textura arenosa, expressam com maior frequência, os efeitos da compactação sobre o desenvolvimento das plantas (Kunz et al., 2013). Essa compactação do solo provoca, além da redução do desenvolvimento radicular, redução na infiltração de água no perfil e consequente aumento no escoamento superficial (Debiasi e Franchini, 2012), podendo resultar em processos erosivos. Entretanto, esse aumento da densidade do solo é limitado às suas camadas mais superficiais, podendo ser temporário e reversível (Cassol, 2003).

0 emprego de técnicas de agricultura de precisão, como sua utilização no manejo localizado do solo, vem sendo amplamente utilizado, melhorando o gerenciamento das atividades agrícolas (Bottega et al., 2013). O estudo da variabilidade espacial dos atributos do solo, por meio da ferramenta geoestatística, pode indicar alternativas de manejo (Montanari et al., 2011). Assim, pode ser minimizada a variabilidade presente nos atributos físicos do solo, permitindo modelar e analisar sua variabilidade espacial para 0 interesse agrícola, podendo ser gerados mapas de variabilidade espacial sem tendência e com variância mínima por meio da técnica de interpolação por krigagem, que é útil para o gerenciamento das ações de manejo do solo.

Diante do exposto, o estudo da variabilidade espacial das características físicas do solo se faz necessário para definir as melhores práticas de manejo do solo em áreas com utilização do sistema de iLP. Assim, o objetivo deste trabalho foi avaliar a variabilidade espacial dos atributos físicos em um solo conduzido com duas coberturas em sistema de iLP.

\section{MATERIAL E MÉTODOS}

O estudo foi desenvolvido na fazenda experimental da Embrapa Arroz e Feijão, em Santo Antônio de Goiás-GO, localizada nas coordenadas geodésicas latitude $16^{\circ} 29^{\prime} \mathrm{S}$ e longitude $49^{\circ} 17^{\prime} \mathrm{W}$. Conforme a classificação de Köppen, o clima da região é classificado como $\mathrm{Aw}$, tropical de savana megatérmico, com duas estações bem definidas, sendo a chuvosa de outubro a abril e a seca de maio a setembro, com precipitação média anual de $1.505 \mathrm{~mm}$. Geologicamente, a região é representada por rochas do complexo granulítico Anápolis-Itauçu, associação de Granulitos Ortoderivados, descritos por Araújo (1994).

O solo utilizado no estudo é um Latossolo Vermelho Acriférrico Típico (EMBRAPA, 2013). Essa ordem de solo apresenta evolução avançada, com atuação expressiva do processo pedogenético de latolização, marcado pela intensa intemperização agindo na transformação dos constituintes minerais primários resultando, na fração argila, um predomínio de minerais secundários como caulinita, óxidos e hidróxidos de ferro e alumínio. Suas principais características descritas foram: horizonte A moderado, horizonte Bw com espessura acima de $150 \mathrm{~cm}$, textura muito argilosa, baixa saturação por bases $(\mathrm{V}<50 \%)$, CTC variando de 9,5 (superfície) a $1,5 \mathrm{cmol}_{\mathrm{c}} \mathrm{kg} \mathrm{arg}^{-1}$ (sub-superfície), razão silte/argila entre 0,36 e 0,13, Ki de 1,68 a 1,31, $\Delta \mathrm{pH}$ de 0 á 0,8 e teor de $\mathrm{Fe}_{2} \mathrm{O}_{3}$ (extraído em $\mathrm{H}_{2} \mathrm{SO}_{4}$ ) entre 19 e 24\% (Santos et al., 2010).

Nas áreas pastejadas, utilizou-se o sistema de pastejo rotacionado, onde os animais entravam quando a pastagem atingia uma altura média de $0,70 \mathrm{~m}$ e eram retirados quando a pastagem atingia 
altura de 0,30 m, com lotação média de 7 unidades animal por hectare.

Para a realização do estudo foi construído um grid amostral regular de $25 \times 25 \mathrm{~m}$, totalizando 50 pontos georeferenciados na área. Em cada ponto foram realizadas três medições de Resistência a Penetração nas duas profundidades e, em seguida, realizada as coletas das amostras indeformadas de solo em duas camadas: P1 (0,00-0,10 m) e P2 (0,20$0,30 \mathrm{~m}$ ), onde se determinou a Umidade gravimétrica e a Densidade do solo.

A coleta das amostras indeformadas foi realizada com auxílio de um amostrador do tipo "Uhland", utilizando cilindros de aço inoxidável com volume aproximado de $100 \mathrm{~cm}^{-3}$. Essas amostras foram envoltas em plástico filme e acondicionadas sob temperatura de aproximadamente $5^{\circ} \mathrm{C}$, até serem processadas no laboratório.

Para a determinação da umidade do solo as amostras foram pesadas e colocadas em estufa a 105 ${ }^{\circ} \mathrm{C}$ até obter peso constante para posterior determinação da umidade à base de massa ou gravimétrica $(\theta \mathrm{g})$ (Embrapa, 1997).

A densidade do solo (Ds) foi calculada pela razão da massa do solo seco e o volume do cilindro, utilizado para coletar as amostras indeformadas (Blake e Hartge, 1986).

A RP do solo foi determinada nas profundidades P1 e P2, utilizando-se um penetrógrafo eletrônico de velocidade constante modelo Falker PLG 5300, seguindo-se conforme normas da ASABE S 313 (ASABE, 2006). Utilizouse um cone com diâmetro de 12,83 mm e ângulo de penetração de $30^{\circ}$. A resolução do equipamento é de $7,7 \mathrm{kPa}$ e 0 índice de cone máximo permitido de $7.700 \mathrm{kPa}$.

Os dados foram avaliados por meio da estatística descritiva, tomando por base as seguintes medidas: média, mediana, mínimo, máximo, desvio padrão, coeficiente assimetria e curtose e o coeficiente de variação, utilizando o software GS+ 7.0 (Gamma Design Software $\circledR$ ).

Para o cálculo dos semivariogramas e seus respectivos ajustes foi utilizado o software geoestatístico GS+, verificando dentre os modelos de semivariogramas fornecidos pelo software (linear, esférico, exponencial e gaussiano), qual melhor se ajustou ao modelo experimental, determinando os valores dos parâmetros: efeito pepita (nugget), patamar (sill) e alcance (range) tendo como base de escolha o maior valor de $\mathrm{R}^{2}$ e a menor Soma de Quadrados do Resíduos (RSS), gerados em cada modelo (Cavalcante et al., 2011; Dalchiavon et al., 2011). Os modelos foram classificados de acordo o grau de dependência espacial (GDE), onde foi calculado pela equação 1, proposta por Dalchiavon e Carvalho (2012) onde classifica o GDE < 20\% como muito baixo, $20 \% \leq \mathrm{GDE}<40 \%$ baixo, $40 \% \leq \mathrm{GDE}<$ $60 \%$ médio, $60 \% \leq \mathrm{GDE}<80 \%$ Alto e $80 \% \leq \mathrm{GDE}<$ $100 \%$ muito alto.

$\mathrm{GDE}=[\mathrm{CO} /(\mathrm{CO}+\mathrm{C})]^{\star 100}$

Onde:

GDE = grau de dependência espacial (Relação de igualdade com os pontos vizinhos);

$\mathrm{CO}=$ efeito pepita;

$C=$ variância estrutural;

$\mathrm{CO}+\mathrm{C}=$ patamar.

Considerou-se ao classificar o GDE de cada atributo de solo avaliado os intervalos de valores propostos pelos autores citados anteriormente. Uma vez verificada a dependência espacial da deposição, através do uso do modelo para análise dos semivariogramas, foram produzidos os mapas de isolinhas utilizando as técnicas da geoestatística de krigagem e co-krigagem ordinária pelo software GS+ 7.0 (Gamma Design Software $囚$ ).

Foram realizadas as análises de correlação de Pearson entre as características físicas do solo e sendo classificados de acordo com adaptação de Figueiredo Filho e Silva Junior (2009). As análises foram realizadas com o auxílio do programa Microsoft Excel, versão 2010. Onde critério de interpretação do índice de correlação linear $(r)$ de Pearson foi de $0,7 \leq r \leq 1,0$ Correlação forte; $0,4 \leq r \leq 0,6$ Correlação Moderada; $0,1 \leq r \leq 0,3$ Correlação fraca e $0,0 \leq r \leq 0,1$ Correlação nula.

\section{RESULTADOS E DISCUSSÃO}

Os resultados referentes à estatística descritiva (Tabela 1) indicaram normalidade dos dados para todas as variáveis estudadas. 
Tabela 1. Estatística descritiva (ED) para os atributos físicos do solo: densidade do solo (Ds), umidade gravimétrica $(\mathrm{Ug})$ e resistência à penetração do solo (RP), nas camadas do solo P1 $(0,00-0,10 \mathrm{~m})$ e P2 (0,20-0,30 m)

\begin{tabular}{|c|c|c|c|c|c|c|}
\hline \multirow[t]{2}{*}{ ED } & \multicolumn{2}{|c|}{$\underset{\left(\mathrm{g} / \mathrm{cm}^{3}\right)}{\text { Ds }}$} & \multicolumn{2}{|c|}{$\frac{U \mathrm{~g}}{(\mathrm{~g} / \mathrm{g})}$} & \multicolumn{2}{|c|}{$\frac{\mathrm{RP}}{(\mathrm{MPa})}$} \\
\hline & $P 1$ & $P 2$ & $P 1$ & $P 2$ & $P 1$ & $P 2$ \\
\hline Média & 1,45 & 1,40 & 0,21 & 0,20 & 2,21 & 2,54 \\
\hline Mediana & 1,44 & 1,41 & 0,24 & 0,266 & 2,17 & 2,53 \\
\hline Desvio padrão & 0,09 & 0,16 & 0,061 & 0,048 & 0,458 & 0,387 \\
\hline Mínimo & 1,29 & 0,41 & 0,098 & 0,10 & 1,19 & 1,56 \\
\hline Máximo & 1,66 & 1,59 & 0,299 & 0,34 & 3,298 & 3,42 \\
\hline Curtose & $-0,76$ & 26,64 & $-1,23$ & 0,07 & $-0,30$ & 0,61 \\
\hline Assimetria & 0,35 & $-4,45$ & $-.0,48$ & $-0,28$ & 0,033 & 0,12 \\
\hline CV (\%) & 6,21 & 11,42 & 29,04 & 24,00 & 20,72 & 15,29 \\
\hline
\end{tabular}

${ }^{*}$ CV- Coeficiente de Variação

A análise descritiva tem por finalidade descrever os dados amostrais por meio de medidas de posição (média e mediana) e de dispersão (variância, desvio padrão e coeficiente de variação) podendo ser utilizada como ferramenta auxiliar para complementar a caracterização do comportamento das variáveis estudadas.

Apesar da ocorrência de algumas distribuições assimétricas e simétricas, os valores da média e mediana de todos os atributos estudados são próximos. Segundo Little e Hills (1978) quando os valores da média e mediana apresentam valores semelhantes, os dados apresentam ou aproximam-se da distribuição normal.

Conforme Cruz et al. (2012), os coeficientes de variação $(\mathrm{CV})$ indicaram baixa variação dos dados $(\mathrm{CV}<10 \%)$, para a densidade na primeira profundidade. A Ds e RP, para a profundidade P2, apresentaram CV médio $(10 \%<\mathrm{CV}<20 \%)$, a Ug apresenta alto coeficiente de variação (Tabela 1), em concordância com os resultados obtidos por Santos et al. (2012) em um Latossolo vermelho distroférrico típico e Schaffrath et al. (2008) em áreas cultivadas sob sistema de plantio direto

Os valores médios da Ds apresentaramse entre $1,45 \mathrm{~kg} \mathrm{dm}^{-3}$ (P1) e $1,40 \mathrm{~kg} \mathrm{dm}^{-3}$ (P2), diminuindo conforme a profundidade, não seguindo a mesma tendência da RP que obteve valores médios entre 2,21MPa (P1) e 2,54 MPa (P2). Nas duas camada 0 valor médio da RP foi superior ao valor de $2 \mathrm{Mpa}$ que é considerado crítico para 0 adequado desenvolvimento das plantas (SILVA, 2008; COLLARES et al., 2008). Carvalho et al. (2006) não encontraram restrição na produtividade de grãos de feijoeiro com a resistência à penetração variando entre 1,290 e 2,870 $\mathrm{MPa}$. Os resultados desse atributo são semelhantes aos encontrados por Martins et al. (2009) e Montanari et al. (2012) em um Latossolo Vermelho Distrófico típico argiloso.

Stefanoski et al. (2013) em um sistema de integração lavora pecuária observou que a presença do gado foi responsável pelo aumento da densidade do solo na camada superior do solo, corroborando com os dados deste trabalho, onde foi observado este aumento na camada de $(0-0,10 \mathrm{~m})$.

As correlações lineares de Pearson entre a resistência a penetração e a umidade do solo foram baixas possivelmente pelo número de observações (Tabela 2). Corroborando com os dados encontrados por Souza et al. (2014) onde não encontraram relação significativa entre a umidade e a resistência a penetração do solo em um Argissolo Amarelo. 
Tabela 2. Matriz de correlação dos atributos de Latossolo Vermelho distrófico de Santo Antônio de Goiás, GO.

\begin{tabular}{llrrrrrr}
\hline & & Ds. P1 & U.P1 & RP. P1 & Ds. P2 & U. P2 & RP. P2 \\
\hline Ds. & P1 & 1 & & & & & \\
U. & P1 & $-0.8916^{*}$ & 1 & & & & \\
RP. & P1 & 0.1952 & -0.1705 & 1 & & & \\
Ds. & P2 & 0.2486 & -0.1140 & -0.1988 & 1 & & \\
U. & P2 & $-0.6728^{*}$ & $0.7160^{*}$ & -0.2006 & -0.1402 & 1 & \\
RP. & P2 & 0.2302 & $-0.3322^{*}$ & -0.0045 & $-0.2809^{*}$ & $-0.2843^{*}$ & 1 \\
\hline
\end{tabular}

Legenda: Ds.P1 = Densidade do solo profundidade (0,00 - 0,20); Ds.P2= Densidade do solo profundidade $(0,00-0,20)$; RP.P1= Resistência a penetração profundidade $(0,00$ - 0,20); RP.P2= Resistência a penetração profundidade $(0,00$ $0,20)$; U.P1 = Umidade profundidade $\left(0,00\right.$ - 0,20); U.P1 = Umidade profundidade $(0,00-0,20) ;{ }^{*}$ Significativo a $5 \%$

Os modelos matemáticos com pares significativos da RP em função dos atributos do solo foram formados pela $R P=f(U)$ e $D s=f(R P)$ nas duas profundidades.

Ainda na Tabela 2 o coeficiente de correlação para o par RP P1 x U P1 foi de $-0,17$ e RP P2 x U P2 foi de $-0,28^{*}$ para 0 par Ds. P1 X RP P1 foi de 0,19 . Dessa forma, a equação variou de forma inversa para a umidade e direta para a resistência a penetração. Assim, para o primeiro par que apresentou correlação negativa, pode-se inferir que com 0 aumento da umidade, ocorrerá uma diminuição da resistência à penetração do solo. Já para o segundo par que apresentou correlação positiva pode-se inferir que com o aumento da densidade ocorrerá o mesmo com a resistência a penetração, conforme encontrado por Valadão Junior et al. (2014).

Para os atributos analisados, em sua maioria, foram verificadas estruturas de dependência espacial indicadas pelos semivariogramas simples ajustados aos modelos gaussiano e exponencial. Onde os atributos que se ajustaram melhor ao modelo gaussiano foram Ds P1, U P1, U P2 e RP P1, e ao modelo exponencial foram Ds P2 e RP P2 (Tabela 3).

Tabela 3. Parâmetros dos semivariogramas ajustados para os atributos físicos de Latossolo Vermelho distrófico de Santo Antônio de Goiás, GO.

\begin{tabular}{|c|c|c|c|c|c|c|c|c|}
\hline Parâmetros & \multicolumn{8}{|c|}{ Geoestatística } \\
\hline \multicolumn{9}{|c|}{$y(h)$ simples } \\
\hline & Prof. & Modelo & $\mathrm{C}_{0}$ & $\mathrm{C}_{0}+\mathrm{C} 1$ & $A$ & $\mathrm{R}^{2}$ & RSS & GDE \\
\hline \multirow{2}{*}{ Densidade } & P1 & Gaussiano & 0.0048 & 0.019 & 159.30 & 0.95 & $1.10^{-6}$ & $25 \%$ \\
\hline & $\mathrm{P} 2$ & Exponencial & 0.0007 & 0.0255 & 17.00 & 0.18 & $3.10^{-5}$ & $2,74 \%$ \\
\hline \multirow{2}{*}{ Umidade } & $P 1$ & Gaussiano & 0.0011 & 0.021 & 270.90 & 0.94 & $5.10^{-7}$ & $5,24 \%$ \\
\hline & $P 2$ & Gaussiano & 0.0013 & 0.008 & 239.00 & 0.97 & $5.10^{-8}$ & $16 \%$ \\
\hline \multirow{2}{*}{$\mathrm{RP}$} & $\mathrm{P} 1$ & Gaussiano & 0.012 & 0.207 & 25.50 & 0.56 & $1.10^{-3}$ & $5,8 \%$ \\
\hline & $P 2$ & Exponencial & 0.083 & 0.202 & 78.60 & 0.87 & $3.10^{-4}$ & $41 \%$ \\
\hline \multicolumn{9}{|c|}{$\mathrm{y}(\mathrm{h})$ cruzado } \\
\hline \multirow{2}{*}{$R P=f(U)$} & P1 & Gaussiano & -0.0001 & -0.0064 & 32.20 & 0.72 & $1.10^{-7}$ & $0,15 \%$ \\
\hline & P2 & Gaussiano & -0.00001 & -0.008 & 76.60 & 0.75 & $2.10^{-5}$ & $0.12 \%$ \\
\hline \multirow{2}{*}{$D S .=f(R P)$} & P1 & Esférico & 0.0002 & 0.0082 & 57.30 & 0.03 & $1.10^{-4}$ & $2,4 \%$ \\
\hline & P2 & Exponencial & -0.0068 & -0.0795 & 510.90 & 0.46 & $2.10^{-4}$ & $8,5 \%$ \\
\hline Densidade & $P 1=f(P 2)$ & Exponencial & 0.0001 & 0.0049 & 17.90 & 0.75 & $1.10^{-7}$ & $2,04 \%$ \\
\hline Umidade & $P 1=f(P 2)$ & Gaussiano & 0.0003 & 0.0046 & 129.80 & 0.94 & $6.10^{-7}$ & $6,5 \%$ \\
\hline $\mathrm{RP}$ & $P 1=f(P 2)$ & EPP & - & - & - & - & - & $100 \%$ \\
\hline
\end{tabular}

$\mathrm{DS}, \mathrm{U}, \mathrm{RP}, \mathrm{DP}, \mathrm{P} 1$ e P2, são respectivamente as densidades do solo, umidade e resistência à penetração, coletados nas camadas do solo; EPP= efeito pepita puro;RSS = soma dos quadrados dos resíduos; GDE = Grau de dependência espacial. 
Figura 1. Componentes geoestatísticos dos atributos físicos do solo em cada profundidade de coleta do solo.

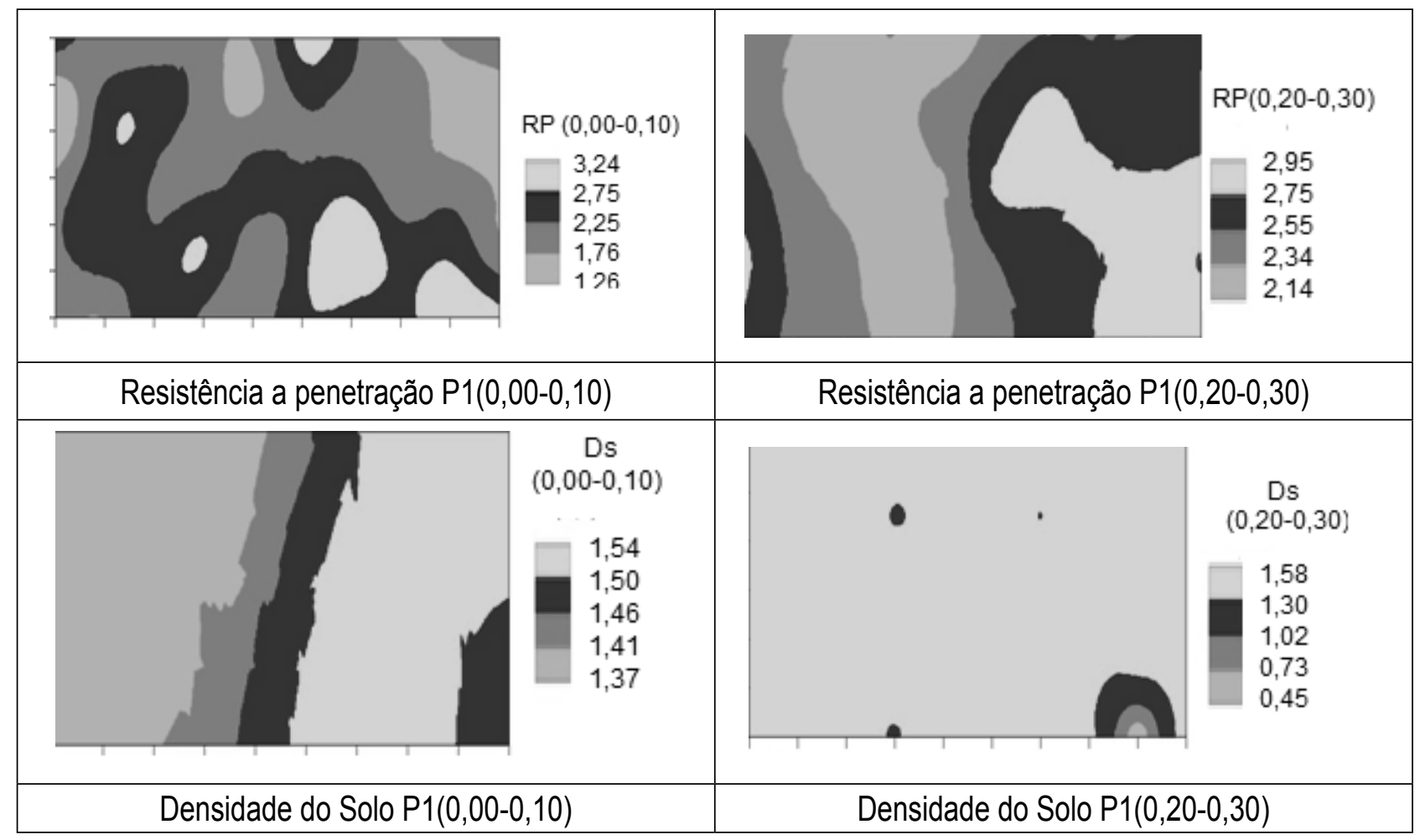

Figura 2. Componentes geoestatísticos dos atributos físicos do solo em função das profundidades de coleta do solo

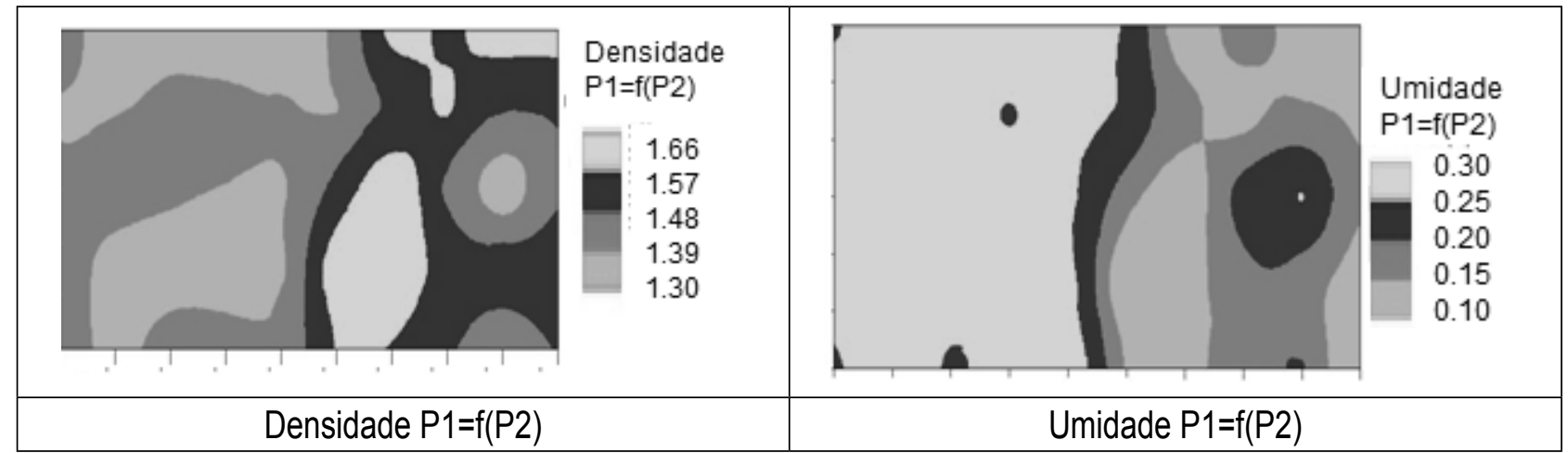

Figura 3. Componentes geoestatísticos da resistência à penetração em função da umididade do solo

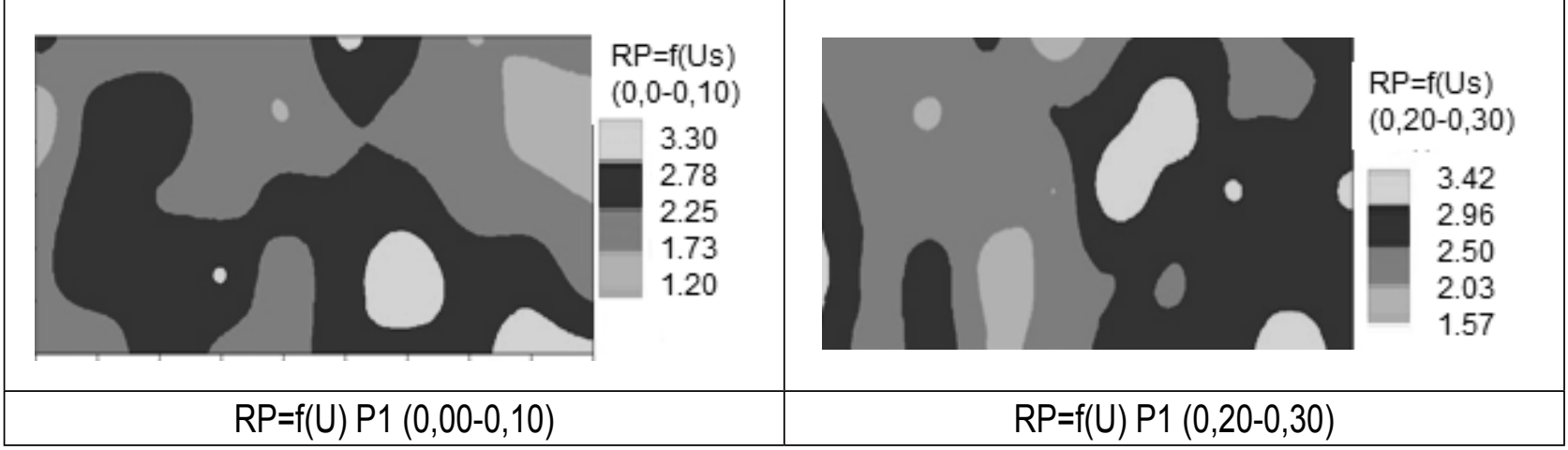

No âmbito da correlação entre atributos do solo $x$ solo, os modelos matemáticos resultaram nas seguintes co-krigagens: RP P1=f(U P1), RP P2 $=f(U P 2)$, Ds $P 1 .=f(R P P 1), D s P 2=f(R P P 2)$ e profundidade para os três atributos $P 1=f(P 2)$, cujos coeficientes de determi- nação espacial $R^{2}$ variaram entre 0,03 e 0,94, equivalentes à variação do GDE entre 0,15 e 8,5\% seguindo a classificação proposta por Dalchiavon e Carvalho (2012) como muito baixo e valores do GDE com valor igual a $100 \%$, classificado como efeito pepita puro. 
Os melhores parâmetros resultantes das co-krigagem foram da P1=f(P2) quando fez a análise da umidade e densidade em relação às duas profundidades onde os coeficientes de determinação espacial foram de 0,94 e 0,75, respectivamente, com alcances de 129,80 e 17,90 m. Em trabalho semelhante Montanari et al. (2010) constataram que apenas a umidade $(U)$ resultou em co-krigagem, cujo coeficiente de determinação espacial $\left(r^{2}\right)$ foi de 0,63 , equivalente ao GDE de 61,6\%.

Conclui-se que o coeficiente de correlação para os pares RP P1 x U P1, RP P2 x U P2 e Ds P1 $X$ RP P1 foram classificados como baixos segundo adaptação de Figueiredo Filho e Silva Junior (2009); dos atributos analisados, em sua maioria, foi verificado uma estrutura de dependência espacial pelos semivariogramas simples ajustados ao modelo Gaussiano e exponencial, onde os ajustados ao modelo gaussiano foram Ds P1, U P1, U P2 e RP P1, e ao modelo exponencial foram Ds P2 e RP P2; no âmbito da correlação espacial entre atributos do solo $x$ solo, os modelos matemáticos resultaram na classificação de índice de dependência espacial como muito baixo e Efeito pepita puro.

\section{REFERÊNCIAS BIBLIOGRÁFICAS}

Alvarenga, R.C. Integração Lavoura-Pecuária. Sete Lagoas: Embrapa Milho e Sorgo, 2005. 16 p.

ASABE - American Society of Agricultural and Biological Engineers. Soil cone penetrometer. ASABE Standard S313.2, St. Joseph, p.903-904, 2006.

Bachmaier, M.; Backes, M. Variogram or semivariogram Understanding the variances in a variogram. PrecisionAgriculture, 2008, 9, 3, 173-175.

Baptista, R.B. Impacto do sistema de integração lavoura pecuária nos estoques de carbono e nitrogênio do solo no cerrado de Goiânia-GO. Santo Antônio de Goiás: EMBRAPAArroz e Feijão, 2012.

Bottega, E.L.; Queiroz, D.M.; Pinto, F.A.C.; Souza, C.M.A. Variabilidade espacial de atributos do solo em sistema de semeadura direta com rotação de culturas no cerrado brasileiro. Revista Ciência Agronômica, 2013, 44, 1, 1- 9.
Brandão, V.S.; Cecilio, R.A.; Pruski, F.F.; Silva, D.D. Infiltração da água no solo. Viçosa: UFV, 3.ed. 2006. $120 f$.

Camargo, O.A.; Alleoni, L.R.F. Compactação do solo e 0 desenvolvimento das plantas. Piracicaba: Escola Superior de Agricultura Luiz de Queiroz, 1997. $132 f$.

Cambardella, C.A.; Moorman, T.B.; Novak, J.M.; Parkin, T.B.; Karlen, D.L.; Turco, R.F.; Konopka, A.E. Fieldscalevariabilityofsoilproperties in central lowasoils. Soils Science Society of America Journal, 1994, 58, 1501-1511.

Cassol L.C. Relações solo-planta-animal num sistema de integração lavoura-pecuária em semeadura direta com calcário na superfície. 2003. 143f. Tese (Doutorado) Universidade Federal do Rio Grande do Sul, Porto Alegre.

Cavalcante, E.G.S.; Alves, M.C.; Souza, Z.M.; Pereira, G. T. Variabilidade espacial de atributos físicos do solo sob diferentes usos e manejos. Revista Brasileira de Engenharia Agrícola e Ambiental, 2011, 15, 3, 237-243.

Coelho, A.M. Agricultura de precisão: manejo da variabilidade espacial e temporal dos solos e da cultura. In: CURI, N. et al. (Ed.). Tópicos em ciência do solo, Sociedade Brasileira de Ciência do Solo, 2003, 249-290.

Costa, N.R.; Andreotti, M.; Bergamasschine, A.F.; Lopes, K.S.M.; Lima, A.E.S. Custo da produção de silagens em sistemas de integração lavoura-pecuária sob plantio direto. Revista Ceres, 2015, 62, 1, 09-19.

Cruz, E.A.; Moreira, G.R.; Paula, M.O.; Oliveira, A.C.M. Coeficiente de variação como medida de precisão em experimentos com tomate em ambiente protegido. Enciclopédia Biosfera, 2012, 8, 14, 220-233.

Cunha, E.Q.; Stone, L.F.; Ferreira, E.P.B.; Didonet, A.D.; Moreira, J.A. Atributos físicos, químicos e biológicos de solo sob produção orgânica impactados por sistemas de cultivo. Revista Brasileira de Engenharia Agrícola e Ambiental, 2012, 16, 1, 56-63.

Dalchiavon, F.C.; Carvalho, M.P.; Nogueira, D.C.; Romano, D.; Abrantes, F. L.; Assis, J.T.; Oliveira, M.S. Produtividade da soja e resistência mecânica à penetração do solo sob sistema plantio direto no cerrado brasileiro. Pesquisa Agropecuária Tropical, 2011, 41, 1, 8-19. 
Debiasi, H.; Franchini, J.C. Atributos físicos do solo e produtividade da soja em sistema de integração lavoura-pecuária com braquiária e soja. Ciência Rural, 2012, 42, 1180-1186.

Derpsch, R.; Roth, C.H.; Sidiras, N.; Kopke, U. Controle da erosão no Paraná, Brasil: Sistemas de cobertura do solo, plantio direto e preparo conservacionista do solo. Eschborn, GTZ, 272f ,1991.

Duarte, G.S. Manejo da altura da pastagem de inverno em sistema integrado lavoura: pecuária com plantio. 2013. 56f. Dissertação (Mestrado em Zootecnia) Faculdade de Agronomia Eliseu Maciel, UFP, Pelotas.

EMBRAPA. Centro Nacional de Pesquisa de Solos (Rio de Janeiro - RJ). Manual de métodos de análise de solo. 2.ed. Revista Atual, Rio de Janeiro, 212f , 1997.

Faraco, M.A.; Uribe-Opazo, M.A.; Silva, E. A. A.; Johann, J.A.; Borssoi, J.A. Seleção de modelos de variabilidade espacial para elaboração de mapas temáticos de atributos físicos do solo e produtividade da soja. Revista Brasileira de Ciências do Solo, 2008, 32, 463-476.

Faroni, C.E.; Trivelin, P.C.O. Quantificação de raízes metabolicamente ativas de cana-de-açúcar. Pesquisa Agropecuária Brasileira, 2006, 41, 6, 1007-1013.

Figueiredo Filho, D.B.; Silva Junior, J.A. Desvendando os mistérios do Coeficiente de Correlação de Pearson $(r)^{*}$. Revista Política Hoje, 2009, 18, 1, 115-146.

Figueiredo, C.C.; Santos, G.G.; Pereira, S.; Nascimento, J.L.; Alves Júnior, J. Propriedades físico-hídricas em Latossolo do Cerrado sob diferentes sistemas de manejo. Revista Brasileira de Engenharia Agrícola e Ambiental, 2009, 13, 146-151.

Isaaks, E.H.; Srivastava, R.M. An introduction to applied geostatistics. New York: Oxford University Press, 1989.561p.

Kluthcouski, J.; Yokoyama, L.P. Opções de integração lavoura-pecuária. In: Kluthcouski, J.; Stone, L.F.; Aidar, H. Integração lavoura-pecuária.1.ed. Santo Antonio de Goiás: Embrapa Arroz e Feijão, 131-141, 2003.

Kunz, M.; Gonçalves, A.D. M.A.; Reichert, J.M.; Guimarães, R.M.; Reinert, D.J.; Rodrigues, M.F.
Compactação do solo na integração soja-pecuária de leite em latosolo argiloso com semeadura direta e escarificada. Revista Brasileira de Ciências Solo, 2013, 37, 1699-1708.

Little, T.M.; Hills, F.J. Agricultural experimentation: design andanalysis. New York: J. Wiley, 1978. 350p.

Maluf, H.J.G.M.; Campos, D.S. Variabilidade espacial da resistência do solo à penetração avaliada ao longo de um cultivo de milho. Revista Agrogeoambiental, 2012, 4,1, 67-72.

Matheron, G. Principles of geostatistics. Economic Geology, 1963, 58, 11246-66.

Mercante, E.; Uribe-Opazo, M.A.; Johann, J.A.; Souza, E.G. Variabilidade temporal da produtividade da soja em uma área experimental com e sem manejo químico localizado utilizando semivariogramas escalonados. In:CONGRESSO BRASILEIRO DE AGRICULTURA DE PRECISÃO, 2004. Piracicaba, SP. Anais... Piracicaba: ESALQ/USP, 2004.

Montanari, R.; Lima, R.C.; Bonini, A.S.; Marques, L.S.; Minguini, R.; Carvalho, M.P.; Ferreiro, J.; Costa, N.R. Variabilidade dos atributos de um Latossolo Vermelho sob plantio direto no cerrado brasileiro e produtividade da soja. Cadernos Laboratorio Xeolóxico de Laxe, 2011, 36, 61-78.

Montanari, R.; Carvalho, M.P.;Andreotti, M.; Dlachiavon, F.C.; Lovera, L.H.; Honorato, M.A.O. Aspectos da produtividade do feijão correlacionados com atributos físicos do solo sob elevado nível tecnológico de manejo. Revista Brasileira de Ciência do Solo, 2010, $34,6,1811-1822$.

Sales, L.E.O.; Carneiro, M.A.C.; Severiano, E.C.; Oliveira, G.C.; Ferreira, M.M. Qualidade física de Neossolo Quartzarênico submetido a diferentes sistemas de uso agrícola. Ciência e Agrotecnologia, 2010, 34, 667-674.

Santos, D.; Souza, E.G.; Nóblega, L.H.P.; Bazzi, C.; Júnior, A.G. Variabilidade espacial de atributos físicos de um Latossolo Vermelho após cultivo de soja. Revista Brasileira de Engenharia Agrícola e Ambiental, 2012, $16,8,843-848$. 
Schaffrath, V.R.; Tormena, C.A.; Gonçalves, A.C.A.; Fidalski, J. Variabilidade e correlação espacial de propriedades físicas de solo sob plantio direto e preparo convencional. Revista Brasileira de Ciência do Solo, 2008, 32, 1369-1377.

Secco. D.; Reinert. D.J.; Reichert. J.M.; Daros. C.O. Produtividade de soja e propriedades físicas de um Latossolo submetido a sistemas de manejo e compactação. Revista Brasileira de Ciência do Solo, 2004, 28, 797-804.

Secco, D.; Daros, C.O.; Secco, J.K.; Fiorin, J.E. Atributos físicos e produtividade de culturas em um Latossolo Vermelho argiloso sob diferentes sistemas de manejo. Revista Brasileira de Ciência do Solo, 2005, 29, 3, 407-414.

Souza, E.B.; Patrocínio Filho, A.P.P.; Pimenta, W.A.; Nagahama, H.J.; Cortez, J.W. Resistência mecânica do solo à penetração em função da sua umidade e do tipo de penetrômetro. Revista Engenharia na agricultura, 2014, 22.

Souza, F.R.; Rosa Junior, E.J.; Fietz, C.R.; Pellin, D.M.P.; Bergamin, A.C.; Gelain, E.; Rosa, Y.B.C.J.; Morphology and stability of aggregates of an Oxisol according to tillage system and gypsum application. Revista Ceres, 2012, 59, 6, 859-866.

Souza, Z.M.; Marques, J.J.; Cooper, M.; Pereira G.T. Micromorfologia do solo e sua relação com atributos físicos e hídricos. Pesquisa Agropecuária Brasileira, 2006, 41, 487-492.

Spera, S.T.; Santos, H.P.; Fontaneli, R.S.; Tomm, G.O. Integração lavoura e pecuária e os atributos físicos de solo manejado sob sistema plantio direto. Revista Brasileira de Ciências do Solo, 2009, 33, 129-136.

Stefanoski, D.C.; Santos, G.G.; Marchão, R.L.; Petter, F.A.; Pacheco, L.P. Uso e manejo do solo e seus impactos sobre a qualidade física. Revista Brasileira de Engenharia Agrícola e Ambiental, 2013, 17, 12, 1301-1309.
Stone, L F.; Silveira, P.M. Efeitos do sistema de preparo e da rotação de culturas na porosidade e densidade do solo. Revista Brasileira de Ciência do Solo, 2001, 25, 395-401.

Tormena, C.A.; Silva, A.P.; Libardi, P.L. Caracterização do intervalo hídrico ótimo de um Latossolo Roxo sob plantio direto. Revista Brasileira de Ciência do Solo, 1998, 22, 573-581.

Topp. G.C.; Zebchuk. W. The determination of soil water desorption curves for soil cores. Canadian Journal of Soil Science, 1979, 59, 19-26.

Vasconcelos A.C.M.; Casagrande, A.A. Fisiologia do sistema radicular In: Dinardo-Miranda, L.L.; Vasconcelos, A.C.M.; Landell, M.G.A. Cana-de-açúcar. Campinas: Instituto Agronômico e Fundação - IAC. Cap.4, p.79-98, 2008.

Vasconcelos, A.C.M; Garcia, J.C. Cana-de-açúcar: ambientes de produção. Informações Agronômicas, $2005,1,110,1-5$.

Valadão Junior, D.D.; Biachini, A.; Valadão, F.C.; Rosa, R.P. Penetration resistance according to penetration rate, cone base sizeand different soil conditions. Journal of Plant Nutrition and Soil Science, 2014, 73, 2, 171-177.

Vilela, L.; Barcellos, A.O.; Martha Júnior, G.B. Plantio direto de pastagens. In: SIMPÓSIO SOBRE MANEJO DA PASTAGEM, 2006. Piracicaba. Anais... Piracicaba, FE-ALQ. 2006. 165-185.

Vilela, L.; Martha,G.B.J.; Macedo, M.C.M.; Marchão, R.L. Júnior, R.G.; Pulrolnik, K.; Maciel, G.A. Sistemas de integração lavoura-pecuária na região do Cerrado. Pesquisa agropecuária brasileira, 2011, 46, 10, 1127-1138.

Zimback, C.R.L. Análise espacial de atributos químicos de solos para fins de mapeamento da fertilidade do solo. 114 f. Tese (Livre-Docência), Faculdade de Ciências Agronômicas-USP, Botucatu, 2001. 